\title{
Missed Diagnosis
}

\section{Cervical spondylotic myelopathy with occult foramen magnum meningioma - a case for MRI?}

\author{
K. Chakravarty, D.G.I. Scott, 'D. Dick and ${ }^{2}$ J. Visick \\ Department of Rheumatology, ${ }^{1}$ Department of Neuromedicine, ${ }^{2}$ Department of Radiology, Norfolk \& \\ Norwich Hospital, Norwich, UK
}

\begin{abstract}
Summary: A fatal case of occult foramen magnum meningioma is described which presented with features of cervical myelopathy confirmed by cervical myelogram. Surgical decompression led to initial improvement followed by progressive deterioration and death. Postmortem examination revealed a $4 \mathrm{~cm}$ diameter foramen magnum meningioma. The dangers of using only standard radiographic investigations of the cervical cord are discussed.
\end{abstract}

\section{Introduction}

Tumours of the foramen magnum often present with ill-defined symptoms mimicking multiple sclerosis, syringeomyelia, normal pressure hydrocephalus and degenerative diseases of the spinal cord. ${ }^{1}$ We describe a patient with a benign tumour of the foramen magnum who presented with features of cervical myelopathy with myelographic evidence of cord compression especially at $\mathrm{C} 5$ and C6.

\section{Case report}

A 76 years old, previously fit woman presented with a 10 week history of coldness and numbness of her hands. She also admitted to progressive lower limb stiffness and an unsteady gait. She had no headaches, sickness or vomiting and no visual, bladder or bowel disturbances. Physical examination revealed distal upper limb weakness and impaired joint position sense in her hands with mild symmetrical paraparesis. Cranial nerve and fundal examination was normal.

Investigations revealed a haemoglobin of 12.1 $\mathrm{g} / \mathrm{dl}$, white cell count $4.6 \times 10^{9} / 1$ with normal platelet count and erythrocyte sedimentation rate. The following investigations were normal or

Correspondence: K. Chakravarty, M.B., M.R.C.P., Rheumatology Research Unit, E6, Addenbrooke's Hospital, Cambridge CB2 2QQ, UK.

Accepted: 20 January 1993 negative: urea and electrolytes, liver and thyrodi function tests, bone chemistry, syphilis serolog,$\vec{\oplus}$ serum $B_{12}$ and red cell folate. The antinuclear factôi was negative but anti-DNA antibody was raised at 94 IU/l (NR < 50 IU/l). Rheumatoid factor, complement levels, creatine kinase and antibody to $\bar{\partial}$ extractable nuclear antigens were within the normal range.

A preoperative cervical spine $X$-ray showed $\stackrel{2}{\Rightarrow}$ severe degenerative changes at $\mathrm{C} 5 / 6$ and $\mathrm{C} 6 / 7 \frac{\mathrm{O}}{3}$ (Figure 1). The chest radiograph and the electrocardiogram were normal. A cervical myelogramo (Figure 2 and 3 ) revealed marked indentation of the cord between $\mathrm{C} 5 / 6$ and $\mathrm{C} 6 / 7$ due to large 3 . osteophytes suggesting considerable cord compres- $\dot{0}$ sion. Cerebrospinal fluid analysis was normal 3 . except for a slightly raised protein of $0.75 \mathrm{~g} / \mathrm{l}(\mathrm{NR} \dot{\mathrm{\delta}}$ $0.10-0.40$ ).

She underwent cervical laminectomy and 0 decompression with good response apart from some initial postoperative dysphagia, thought to be due to delay in the lift of the larnyx as a result of $\underset{N}{ }$ possible damage to $\mathrm{C} 3,4$ and 5 nerve fibres. Her . dysphagia later improved allowing her to swallow $N$ food normally.

However, 6 weeks postoperatively, her dys- 0 phagia returned and she became progressively weak and immobile with episodes of choking. Her $\frac{}{\varnothing}$ clinical course was of rapid and progressive $\stackrel{\oplus}{\rightarrow}$ deterioration and she died within 10 days.

A postmortem examination revealed a $4 \mathrm{~cm}$ firm $\stackrel{0}{\circ}$ tumour at the foramen magnum in front of the 
a

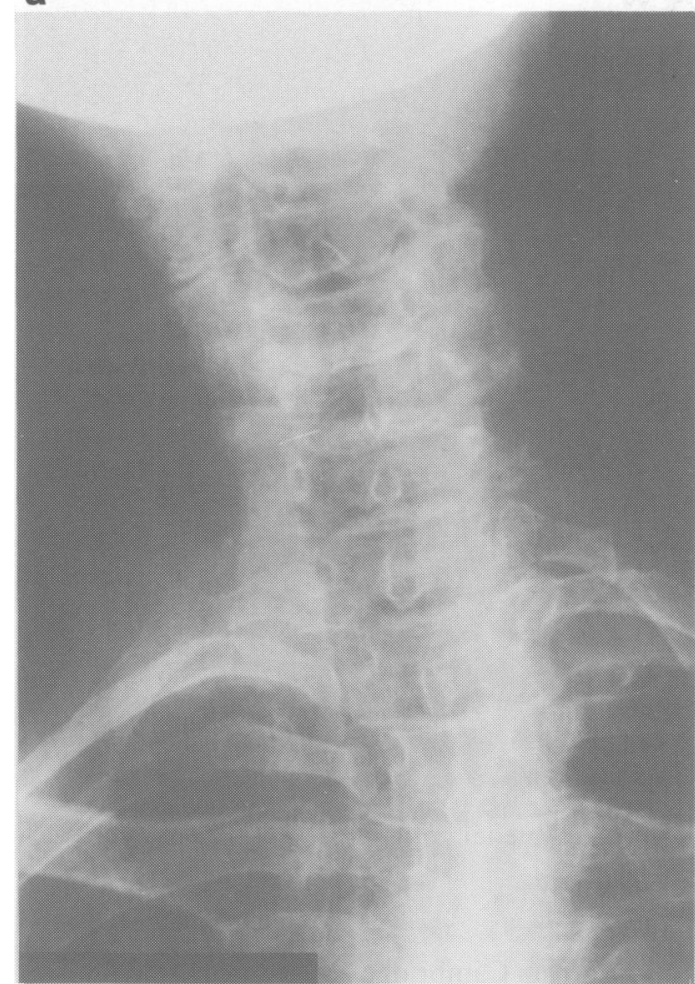

b

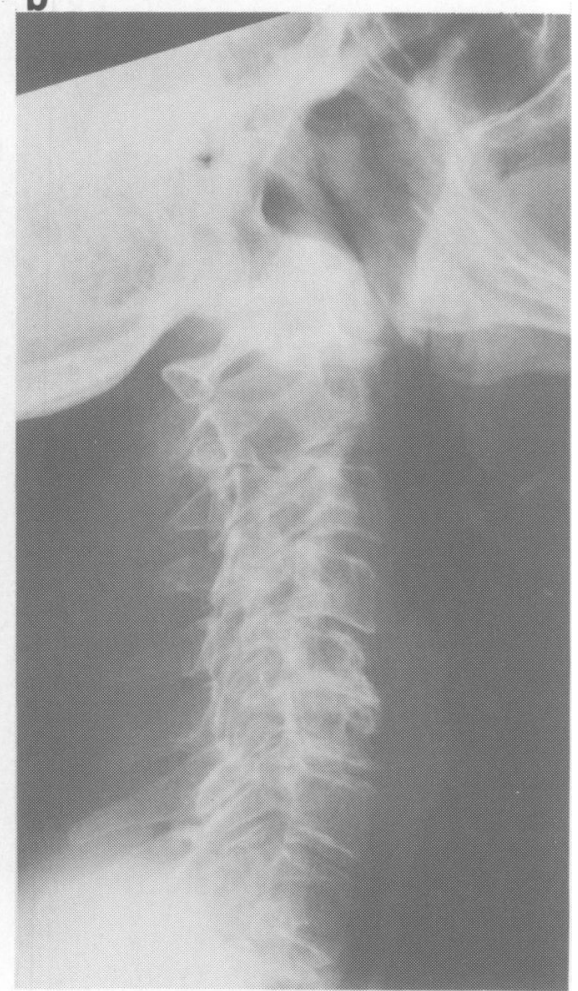

Figure 1 Preoperative X-ray of cervical spine showing severe degenerative changes. (a) Anterior-posterior, (b) lateral.

medulla oblongata and the histology confirmed a meningioma. The spinal cord was atrophic with a distal area of infarction in the upper thoracic region. The cervical spinal cord appeared unremarkable.

\section{Discussion}

Our patient illustrates the problems associated with the diagnosis of benign tumours at the foramen magnum, particularly in the presence of cervical spondylosis. Cervical myelography gave no specific information above the lower cervical cord and in retrospect was potentially dangerous because of the risk of coning.

The clinical presentation of foramen magnum meningioma is varied and neurological examination rarely demonstrates signs indicating the exact localization of the pathology. In one series $66 \%$ of patients presented with only neck pain, often in the C2 nerve root distribution, $49 \%$ with gait disturbances, $22 \%$ with bladder dysfunction and only $12 \%$ with dysphagia. ${ }^{1}$ Our patient developed pronounced dysphagia in the immediate postoperative period followed by gradual improvement, only to recur as a preterminal event. It is possible that the severe post-surgical dysphagia was related to tissue oedema adjacent to the primary lesion associated with increase in cerebrospinal fluid pressure which improved with wound healing. The late relapse probably reflected progressive herniation of the tumour into the foramen magnum resulting in coning and death.

Our case highlights the risks of standard investigative procedures such as cervical myelography in elderly patients with clinical features of cervical myelopathy. Cervical myelography is essentially an invasive in-patient procedure and requires considerable time and experience of the radiologist with its attendant risks, as evident in our case. In one study computerized tomography (CT) scanning with intravenous contrast was diagnostic in $75 \%$ of cases. Another study demonstrated that metrizamide myelography and CT scanning was more informative than myelography alone. ${ }^{2}$ However, myelographic demonstration of the tumour requires complete contrast filling of all portions of the subarachnoid space, requiring more time than a 'routine' procedure. CT scanning is marginally superior to the myelography but the medulla and the cervical spinal cord are virtually never shown 


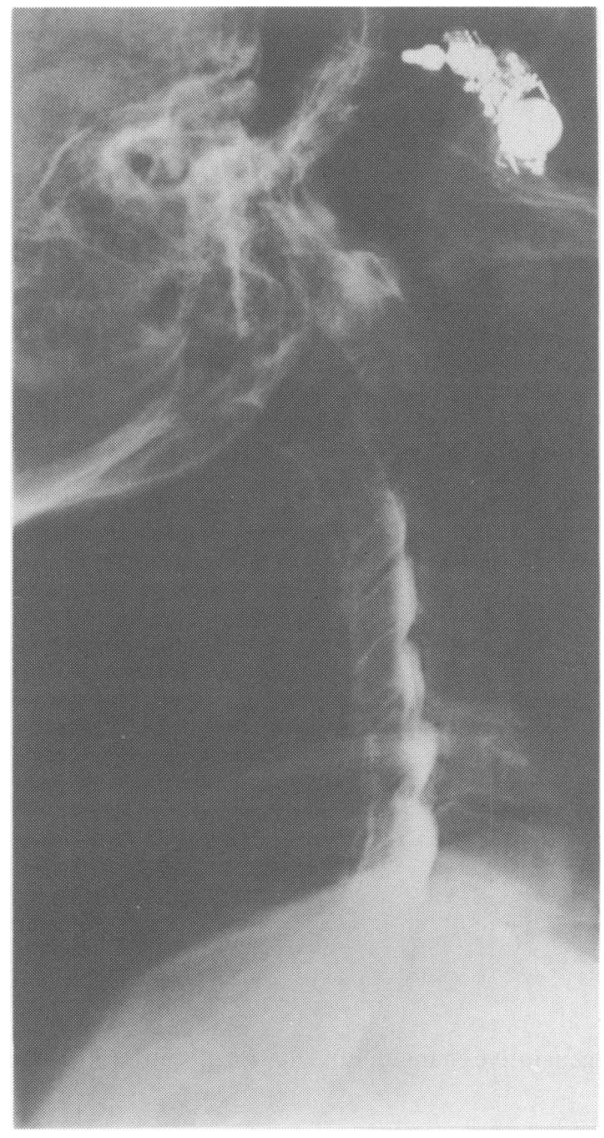

Figure 2 X-ray (lateral-cervical myelogram) showing severe compression at $\mathrm{C} 5 / 6$.

well in absence of bony changes, calcification or alteration with contrast enhancement. ${ }^{3}$ Previous studies have recommended magnetic resonance imaging (MRI) as the most accurate noninvasive and cost-effective way of evaluating abnormalities of the cranio-cervical junction. ${ }^{4-12}$

A recent survey from a regional neurological centre has shown a significant change in diagnosis $(20 \%)$ and management $(27 \%)$ following MRI scan. ${ }^{13}$ It was also shown to be cost effective if other radiographic procedures could be reduced in the run up to MRI with a slightly higher risk of inappropriate request for MRI scans. A similar

\section{References}

1. Meyer, F.B., Edersold, M.J. \& Reese, D.F. Benign tumours of the foramen magnum. $J$ Neurosurg 1984, 61: 136-142.

2. La-masters, D.L., Watanabe, T.J., Chambers, E.F. et al. Multiplanar metrizamide-enhanced CT scan of the foramen magnum. AJNR 1982, 3: 485-494.

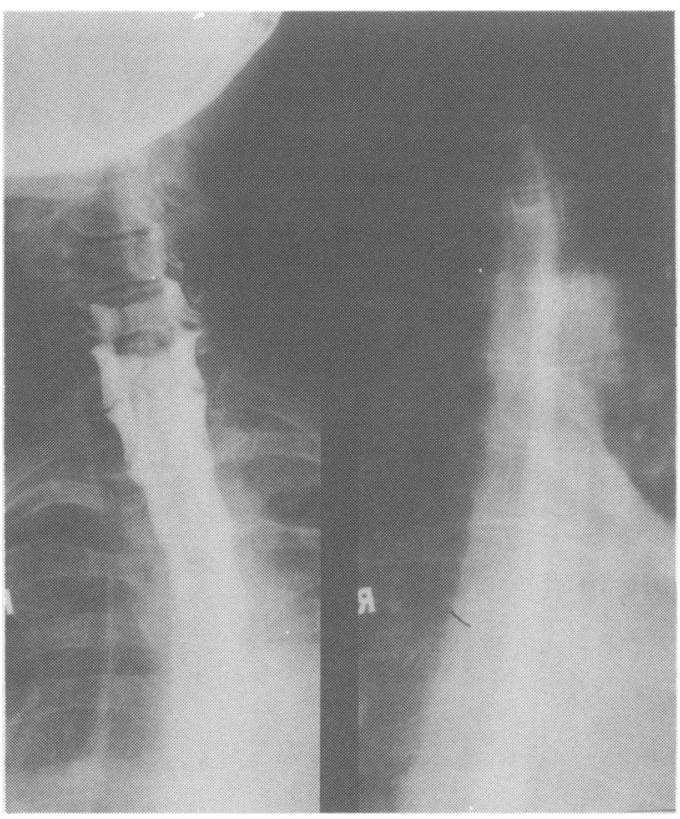

Figure 3 Anterior posterior view of the cervical myelogram.

study ${ }^{14}$ from Cambridge has shown that MRI scan of the neck is less expensive than a CT myelogram which includes in-patient stay for at least 24 hours in uncomplicated cases.

It is clear from our case that MRI scan would have changed the diagnosis and saved the patient from unnecessary surgery. Perhaps a correct diagnosis would have helped her to undergo the appropriate surgery which could have saved her life. It is important to recognize that foramen magnum meningiomas are totally resectable and a good quality of life can be expected postoperatively. MRI scan is clearly the investigation of first choice in patients with cervical myelopathy and should replace other older investigations.

\section{Acknowledgement}

We are grateful to Mr Waters for his help with the surgical management of the patient.

3. Lee, B.C., Michael Deen, D.F., Kneeland, B.J. \& Cahill, T.P. MRI imaging of the cranio-cervical junction. AJNR 1985, 6: 209-213. 
4. Young, I.R., Bails, D.R., Burl, M. et al. Initial clinical evaluation of the whole body nuclear magnetic resonance (NMR) tomograph. J Comput Assist Tomogr 1983, 7: 575-584.

5. Young, I.R., Bailes, D.R., Burl, M. et al. Initial clinical evaluation of the whole body nuclear magnetic resonance (NMR) tomograph. J Comput Assist Tomogr 1982, 6: 118-129.

6. McGinnis, B.D., Brady, T.J., New, P.F.J. et al. Nuclear magnetic resonance (NMR) imaging of tumors in the posterior fossa. J Comput Assist Tomogr 1983, 7: 575-584.

7. Bydder, G.M., Steiner, R.E. Thomas, D.J., Marshall, J., Gilderdale, D.J. \& Young, I.R. Nuclear magnetic resonance imaging of the posterior fossa: 50 cases. Clin Radiol 1983, 20: 173-188.

8. Randell, C.P., Collins, A.G., Young, I.R. et al. Nuclear magnetic resonance imaging of posterior fossa tumors. AJNR 1983, 4: 1027-1034; AJR 1983, 141: 489-496.

9. Lee, B.C.P., Kneeland, B.J., Deck, M.D.F. \& Cahill, P.T. Posterior fossa lesions: magnetic resonance imaging. Radiology 1984, 153: 137-143.
10. Modic, M.T., Weinstein, M.A., Pavlicek, W., Boumphrey, F., Starnes, D. \& Duchesneau, P.M. Magnetic resonance imaging of the cervical spine: technical and clinical observations. AJNR 1984, 5: 15-22; AJR 1983, 141: 1129-1136.

11. Han, J.S., Kaufmann, B., El Yousef, S.J. et al. NMR imaging of the spine. $A J N R$ 1983, 4: 1151-1159; AJR 1983, 141: 1137-1145.

12. Norman, D., Mills, C.M., Brant-Zawadzki, M., Yeates, A., Crooks, L.E. \& Kaufman, L. Magnetic resonance imaging of the spinal cord and canal: potentials and limitations. AJNR 1984, 5: 9-14; AJR 1983, 141: 1147-1152.

13. Szczepura, A.K., Fletcher, J. \& Fitzpatrick, J.D. Costeffectiveness of magnetic resonance imaging in the neurosciences. Br Med J 1991, 303: 1435-1438.

14. Southern, P., Teale, A., Freer, C. et al. An audit of the clinical use of magnetic resonance imaging of the head and spine. Health Trends 1991, 23: 75-79. 